\title{
Dairy producer satisfaction and knowledge transfer with the veterinary- administered risk assessment and management plan in a voluntary Johne's disease control program
}

\author{
Karen A. MacDonald-Phillips, ${ }^{1 *}$ (1) Greg P. Keefe, ${ }^{1} \odot$ John VanLeeuwen, ${ }^{1} \odot$ Darcy H. Shaw, ${ }^{2}$ J. McClure,,${ }^{1} \odot$ \\ and Shawn L. B. McKenna ${ }^{1}$ (D) \\ ${ }^{1}$ Department of Health Management, Atlantic Veterinary College, University of Prince Edward Island, 550 University Avenue, Charlottetown, \\ Prince Edward Island, Canada C1A 4P3 \\ ${ }^{2}$ Department of Companion Animals, Atlantic Veterinary College, University of Prince Edward Island, 550 University Avenue, Charlottetown, \\ Prince Edward Island, Canada C1A 4P3
}

\begin{abstract}
The Atlantic Johne's Disease Initiative (AJDI) aims to control Mycobacterium avium ssp. paratuberculosis infection by using veterinary-administered risk assessments to identify high-risk management practices and prompt changes in management behavior. Objectives for this study were to measure producer satisfaction with the veterinary-administered risk assessment and management plan (RAMP) process in a voluntary Johne's disease (JD) control program, compare RAMP-specific satisfaction results based on herd JD status, and measure knowledge transfer from certified veterinarians to producers during the RAMP. A satisfaction questionnaire was adapted to the RAMP process in the AJDI to measure producer satisfaction. The questionnaire included 9 RAMP-specific producer satisfaction items, 1 global RAMP satisfaction item, and 16 questions to assess producer knowledge and knowledge translation about JD, bovine viral diarrhea (BVD), and bovine leukosis virus (BLV) during the RAMP (BVD and BLV used for comparison purposes). A total of 133 dairy producers in the AJDI (79.6\% response rate) completed the questionnaire by telephone. The RAMP-specific satisfaction was high among the AJDI producers surveyed, and these results were not found to differ based on herd JD status. The lowest satisfaction scores and the highest number of "unable to assess" responses were for the item relating to cost. Factors that contributed to RAMP-specific producer satisfaction were not identified from the demographic and herd information available in this study. The knowledge scores indicated moderate knowledge about JD and fair knowledge about BVD and BLV. Evidence of knowledge translation from the RAMP was mixed in
\end{abstract}

Received April 28, 2021

Accepted October 15, 2021.

*Corresponding author: kamphillips@hotmail.ca this study. Bovine viral diarrhea knowledge scores were not found to differ based on whether or not the certified veterinarian discussed BVD during the preceding RAMP, but BLV knowledge scores were higher among dairy producers that discussed BLV during the preceding RAMP. Strengths and gaps in producer knowledge about these 3 infectious diseases were identified. By using this producer questionnaire, interventions aimed at improving the content, delivery, and satisfaction of RAMP in JD control programs, such as the AJDI, can be developed.

Key words: satisfaction, risk assessment and management plan, Johne's disease, knowledge transfer, infectious disease

\section{INTRODUCTION}

Prevention and management of infectious diseases of dairy cattle are important for the production of dairy products that are safe, nutritious, and meet high consumer expectations. Johne's disease (JD), also known as paratuberculosis, is an incurable infectious disease of ruminants that affects dairy herds around the world. It is caused by a bacterium, Mycobacterium avium ssp. paratuberculosis (MAP), and results in severe ill thrift due to chronic enteritis (Tiwari et al., 2006). Johne's disease results in substantial financial cost to the Canadian dairy industry (Wolf et al., 2014). Also, research into associations between MAP and Crohn's disease in humans is ongoing (Chiodini et al., 2012; Sweeney et al., 2012; Atreya et al., 2014). Johne's disease has been identified as one of the top disease priorities of Canadian dairy farmers, university researchers, and practicing veterinarians (Bauman et al., 2016).

Control programs for JD have been widely implemented and are largely based on the use of veterinaryadministered risk assessment (RA) to identify highrisk management practices and to prompt changes in management behavior to prevent and reduce JD risk 
on farm (Nielsen, 2007; Collins et al., 2010; Barker et al., 2012). The success of RA-based control programs for JD depends on the veterinarian's ability to communicate with their clients and the producer's adherence with the recommended best management practices (Sorge et al., 2010; Roche et al., 2015). Previous studies that have evaluated RA-based JD control programs have indicated that dairy producers are not necessarily adopting management practices as recommended, particularly with increasing duration of participation in a control program (Ridge et al., 2005; Sorge et al., 2010; Wolf et al., 2015).

To better understand factors that were associated with behavioral change in JD control programs, several studies have described characteristics (i.e., demographic factors, knowledge, and attitudes) of producers and factors as assessed in an RA that motivated producers to make management improvements (Roche et al., 2015; Wolf et al., 2015). To gain additional insight into whether dairy producers are likely to adopt management practices to control JD, it would be useful to know whether they are satisfied with the risk assessment and management plan (RAMP) process.

Satisfaction with care, visits, and appointments is considered to be an important outcome of medical encounters in human and veterinary medicine (Woodcock and Barleggs, 2005; Coe et al., 2010; Shaw et al., 2016). Measuring satisfaction in medicine has become important for several reasons, including to evaluate the quality of health care provided (Sitzia and Wood, 1997; Jackson et al., 2001; Bragadóttir and Reed, 2002), to isolate problem areas in service delivery and generate ideas for solutions (Locker and Dunt, 1978; Jackson et al., 2001), and to improve compliance with health care regimens, changes, and recommendations (Larsen and Rootman, 1976; Bartlett et al., 1984; Bell et al., 2002).

To measure client satisfaction in companion animal practice, 2 questionnaires have been developed and validated, the veterinary service satisfaction questionnaire (VSSQ; Woodcock and Barleggs, 2005) and the client satisfaction questionnaire (CSQ; Coe et al., 2010). The VSSQ is intended to assess client's overall satisfaction with small-animal veterinary services, whereas the CSQ is intended to measure appointment-specific client satisfaction with veterinary care in companion animal practice (Woodcock and Barleggs, 2005; Coe et al., 2010). Coe et al. (2010) demonstrated that the CSQ could be used as an outcome measure of appointment-specific client satisfaction in companion animal practice; however, continued evaluation of the CSQ in different contexts was recommended.

A recent study applied a slightly modified CSQ to dairy farmers [e.g., by exchanging the word "pet(s)" for "animals(s)"] to elicit their satisfaction with veterinary advisors after herd health and production management farm visits (Ritter et al., 2019). Overall, Ritter et al. (2019) reported that farmers were satisfied with their veterinarian's communication, and their satisfaction was positively associated with their preparedness to adopt veterinary advice. Client satisfaction has also been linked to adherence with treatment and management plans in several veterinary studies (Wassink et al., 2010; Kanji et al., 2012; Ritter et al., 2019). Formal research into client satisfaction in food animal production medicine and large animal infectious disease control programs is limited. In the absence of an established instrument to measure satisfaction for food animal purposes, adaptation of the CSQ to measure RAMP-specific client satisfaction may provide valuable insight into whether dairy producers are likely to adopt best management practices to control JD.

The Atlantic Johne's Disease Initiative (AJDI) was established in 2011 as a long-term, voluntary, on-farm, prevention and control program to reduce the prevalence and effect of JD on the dairy herds of Atlantic Canada. A combination of environmental culture herd categorization (EC) and farm-specific veterinaryadministered RAMP were included in the AJDI for the purpose of JD prevalence reduction. Funding was provided by the AJDI for both herd categorizations and veterinary fees to conduct the RAMP. Producer contributions to the RAMP included their time, providing clarifying information to the herd veterinarian during the assessment of JD risk, and working with the herd veterinarian to reach a consensus on management changes to decrease JD risk on their farm. In the AJDI, herd categorization and RAMP completion was done annually. If a herd was categorized as negative through EC (EC-negative) for $2 \mathrm{yr}$ in a row, herd categorization and RAMP completion was then done every other year, as long as they continued to be EC-negative.

The objectives for this study were to measure RAMPspecific producer satisfaction in a JD control program, compare RAMP-specific satisfaction results based on herd JD status, and measure knowledge transfer from certified veterinarians to producers in a JD control program.

\section{MATERIALS AND METHODS}

\section{Source Population and Sampling Method}

The 167 AJDI participant herds who completed a RAMP from July 2013 to March 2014 were invited to participate (the source population) for this study. The farm personnel who completed the RAMP with the certified veterinarians were requested to complete a satisfaction questionnaire by telephone by 1 interviewer 
from January to March 2014. The required sample size was estimated to be 38 herds to estimate the overall satisfaction score to within 0.2 out of 6.0 of the actual value, with $95 \%$ confidence, based on calculations with ProMESA 2.3.0.2 (EpiCentre, IVABS, Massey University, and Instituto Nacional de Tecnología Agropecuaria).

Both written and verbal consent was provided by participants; written consent to participate in the AJDI was obtained at herd enrollment, and verbal consent to participate in the RAMP satisfaction questionnaire was obtained upon initial telephone contact by the interviewer. Animal use protocols (protocol 11-038 file number 6004374) and biosafety permits (file number 6004379) were approved by their respective compliance bodies for this project. Based on the information in those protocols submitted at that time in 2010, research ethics board approval was deemed to be not necessary by the University of Prince Edward Island.

\section{Data Collection}

The 15-item CSQ developed by Coe et al. (2010) was adapted to the veterinary-administered RAMP, creating a 9-item measure of RAMP-specific producer satisfaction and 1 global satisfaction item. The indi- vidual and global satisfaction items were scored using a 6 -point Likert scale with 1 being "poor" and 6 being "excellent." This unbalanced 6-point adjectival format was used in the CSQ as it had a G-coefficient for internal consistency of 0.96 , provided the opportunity for clients to make fine distinctions in their evaluation of satisfaction, and maximized variance in responses for most items (Coe et al., 2010). There was also a response option of "unable to assess." A direct comparison of the items in the CSQ and the producer satisfaction questionnaire and rationale for item exclusion is shown in Table 1. Two items were excluded from the CSQ due to the differences in focus from individual pets to herds. Four other CSQ items were not applicable due to the structure of the RAMP visits as follows: the reason for the visit was predefined to do the RAMP, some items of the RA were to be assessed by certified veterinarians independent of the producer, certified veterinarians were not administering treatments or doing procedures to conduct the RAMP, and the costs of the veterinary fees to conduct the RAMP were paid by AJDI.

To measure knowledge transfer from certified veterinarians to producers during the RAMP, questions about JD were included in the questionnaire. Due to the time period defined for inclusion in the source population, it was likely that most herds that were eligible for inclu-

Table 1. Adaptation of the client satisfaction questionnaire to the producer satisfaction questionnaire for the risk assessment and management plan (RAMP) in the Atlantic Johne's Disease Initiative (AJDI)

Client satisfaction questionnaire item ${ }^{1}$

Your sense of the vet's confidence interacting with you and your pet

The veterinarian's examination of your pet

The veterinarian's discussion of options with you

How well the veterinarian involved you in decisions

The veterinarian's discussion of the cost with you

The interest the veterinarian expressed in your opinion

The amount of information you received from the veterinarian

How well the veterinarian addressed all of your concerns

The amount of time the veterinarian spent with you and your pet

Global satisfaction item

The amount of attention the veterinarian gave your pet

How well the veterinarian understood the reason for your visit

How well the veterinarian involved you in the entire appointment

How well the veterinarian explained treatments and procedures

How well you understood the costs today

The veterinarian's recognition of the role this pet has in your life
RAMP-specific producer satisfaction questionnaire item or exclusion rationale ${ }^{2}$

Your sense of the vet's comfort and confidence interacting with you regarding Johne's disease

How well the vet assessed the Johne's disease risk factors on your farm

Your understanding of the management options for Johne's disease on your

farm

Your level of involvement in the management plan decisions

The vet's discussion of costs with you

The interest the vet expressed in your opinion

The amount of information you received from the vet

How well the vet addressed all of your concerns

The amount of time the vet spent with you

Your overall experience with the vet during the RAMP

NA-Certified veterinarian was assessing herd risks as opposed to focusing on individual animals

NA-Reason for the visit was predefined to do the RAMP

NA-Some items in the risk assessment were to be assessed by the certified veterinarian independently

NA-Certified veterinarian was not administering treatments or doing procedures

NA-The veterinary fees to conduct the RAMPs were paid by AJDI

NA-Certified veterinarian was assessing herd risks as opposed to focusing on individual animals

${ }^{1}$ Coe et al., 2010.

${ }^{2} \mathrm{NA}=$ not applicable (excluded). 
sion in this study would have been responding to the questionnaire based upon their experience with their second RAMP in the AJDI. Therefore, the responses to the JD knowledge questions would represent both knowledge transfer during the preceding RAMP and knowledge about JD that the producers had before the preceding RAMP, possibly from their first RAMP. To increase the likelihood of discussing information novel from previous RAMP and to broaden the focus of RAMP education from the control of JD to other infectious diseases of dairy cattle, certified veterinarians were requested to also discuss bovine viral diarrhea (BVD) and bovine leukosis virus (BLV) during the RAMP conducted from July 2013 onward, for 2 reasons. Clinical experience of the authors suggested that baseline producer knowledge about BVD was low and BLV was moderate. Also, some management practices that decrease the risk of JD introduction to a herd or transmission within a herd are also best management practices (e.g., colostrum or milk pasteurization) to prevent and control BLV in dairy production (LeBlanc et al., 2006; Nekouei et al., 2015b). Two-page information summary sheets about JD, BVD, and BLV were prepared and circulated to the certified veterinarians in June 2013.

Fourteen questions to assess producer knowledge about JD, BVD, and BLV were included in the questionnaire. All questions used a closed format with either multiple choice or yes or no options. A response option of "unable to assess" was also provided for some questions. The specific wording for the knowledge questions and possible responses are included in the Supplemental File (https://data.upei.ca/islandora/object/ researchdata\%3A679; MacDonald-Phillips et al., 2021). To compare infectious disease baseline knowledge with knowledge transfer during the RAMP, the questionnaire also included 2 items to determine if the certified veterinarian discussed BVD or BLV during the preceding RAMP. The appropriateness and understandability of the knowledge questions were assessed by questionnaire pre-test with 3 AJDI herds.

\section{Statistical Analysis}

Questionnaire responses on paper were followed by dual data entry using EpiData 3.1 (EpiData Association). Statistical analysis was completed using Stata 14.2 (StataCorp LLC). For this study, statistical significance was defined as having $P<0.05$. Data about the herds' preceding RAMP (JD herd categorization by EC, RAMP score, and number of RAMP completed) and production data (where available) were combined with the questionnaire data for statistical analyses.
Descriptive statistics were calculated for the demographic and herd data, the RAMP-specific satisfaction items, and the knowledge transfer items. Satisfaction item responses of "unable to assess" were treated as missing values. Knowledge question responses were coded as dichotomous variables, with the values "correct" and "not correct" (responses that were either incorrect or "I'm uncertain"). Shapiro-Wilk test statistics were used to assess normality for the overall satisfaction scores, the global satisfaction scores, and the knowledge scores.

If the Shapiro-Wilk test indicated the assumptions of normality were violated, nonparametric statistical techniques were used for inferential statistics, including Spearman rank correlations and Mann-Whitney U tests. We used $t$-tests if the assumptions of normality were not violated. To identify simple associations between the overall satisfaction score and the demographic and herd information variables, univariable analyses were conducted using linear mixed models with random effects at the veterinarian level. The random effects of veterinarian were included in the models due to the potential for clustering of farms within veterinarians conducting RAMP.

To further evaluate associations between the overall satisfaction score and the demographic and herd information variables, a multivariable linear mixed model with random effects at the veterinarian level was conducted. The univariable analyses described above were used to screen variables (cut-off at $P<0.1$ ) for inclusion into the multivariable model. All variables below the cut-off were initially included in the model, and then backward stepwise elimination of variables that had a Wald test, or multiple Wald tests, with $P>0.05$ was used to determine the final model. Possible 2-way interactions and confounding variables were assessed as well, where relevant, as recommended by Dohoo et al. (2009)

\section{RESULTS}

\section{Demographic and Herd Information}

Of the 167 AJDI herds eligible for the study, $79.6 \%$ responded to the telephone questionnaire $(\mathrm{n}=133$ herds). Demographic information for the participating herds is detailed in Table 2. Most of the questionnaires were administered following the herd's second EC herd categorization $(90.2 \% ; \mathrm{n}=120)$, and $27.1 \%$ were categorized as EC-positive $(\mathrm{n}=36)$. Eighty-seven of the herds were enrolled in a milk-recording program (Valacta). Of the 20 certified veterinarians who conducted RAMP in this study, most were men $(70.0 \%)$. 


\section{RAMP-Specific Producer Satisfaction}

The mean overall satisfaction score, taken as the mean of the 9 individual satisfaction items in the questionnaire, was 5.2 out of 6.0 (95\% CI: 5.1, 5.3). The mean global satisfaction score was 5.6 out of 6.0 (95\% CI: $5.5,5.7)$. The Shapiro-Wilk test statistics for both the overall and global satisfaction scores were significant $(P<0.01$ for both scores $)$, indicating the assumptions of normality were violated. Therefore, nonparametric statistical techniques were used. A positive correlation was detected between the overall satisfaction score and the global satisfaction score $(\mathrm{r}=0.68 ; \mathrm{n}=133 ; P<$ 0.01 ). Significant differences were not detected in either the overall satisfaction score or the global satisfaction score when stratified by herd EC categorization $(P=$ 0.31 and $P=0.88$, respectively).

Table 3 presents descriptive statistics for the global satisfaction item and the 9 individual satisfaction items in the questionnaire. Responses of "unable to assess" were received for 3 of the satisfaction items, specifi- cally, "the vet's discussion of costs with you" $(\mathrm{n}=8)$, "how well the vet addressed all of your concerns" ( $\mathrm{n}=$ 1 ), and "the amount of time the vet spent with you" $(\mathrm{n}=1)$. For 5 of the 9 individual satisfaction items, the mean scores were at least 5.4 out of 6.0 , and all of these items had median scores of 6 . The mean score for the satisfaction item "the vet's discussion of costs with you" was substantially lower than the other items at 4.1 out of 6.0 (95\% CI: 3.9, 4.4), with a substantially higher standard deviation than the other items. The mean score for "your understanding of the management options for JD on your farm" was slightly lower than the other items at 5.0 out of 6.0 (95\% CI: 4.9, 5.2).

During investigation of factors associated with RAMP-specific producer satisfaction, little correlation was detected among satisfaction rating scores within clusters of RAMP done by the same veterinarian. The largest intraclass correlation coefficient calculated from the univariable analyses was 0.02 (95\% CI: 0.00, 0.65). In spite of the low correlation detected statistically, the random effects of veterinarian were retained in the mod-

Table 2. Herd, producer, and certified veterinarian demographic information for the producer satisfaction questionnaire for the risk assessment and management plan (RAMP) for 133 dairy producers in the Atlantic Johne's Disease Initiative

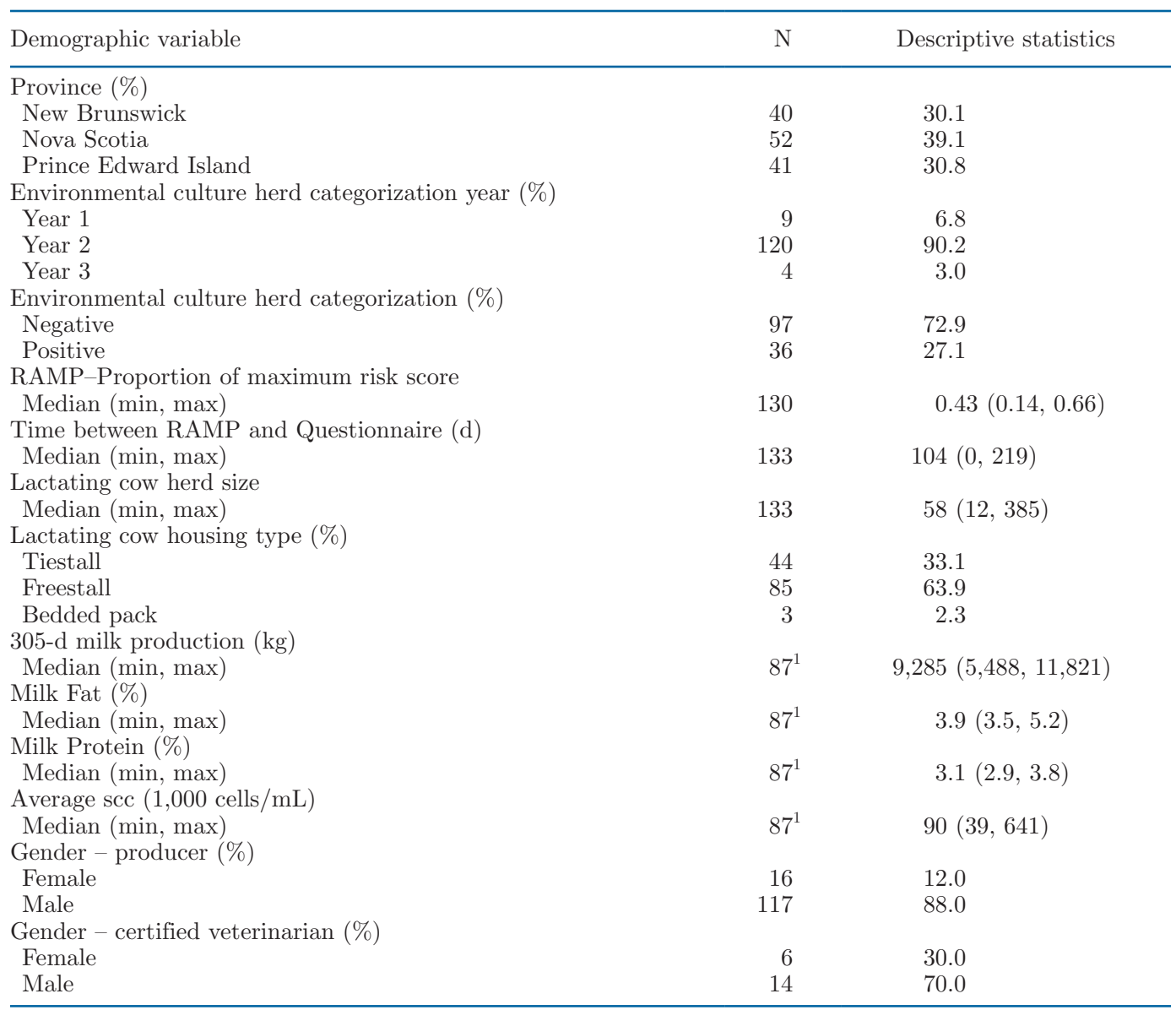

${ }^{1}$ Data only available for 87 herds enrolled in a milk-recording program. 
Table 3. Satisfaction item scores in the producer satisfaction questionnaire for the risk assessment and management plan (RAMP) for 133 dairy producers in the Atlantic Johne's Disease Initiative

\begin{tabular}{|c|c|c|c|c|c|c|}
\hline Satisfaction item ${ }^{1}$ & $\begin{array}{l}\text { Unable } \\
\text { to assess }\end{array}$ & Minimum & Mean & $\mathrm{SD}$ & Median & Maximum \\
\hline $\begin{array}{l}\text { Your sense of the vet's comfort and confidence interacting } \\
\text { with you regarding Johne's disease }\end{array}$ & 0 & 3 & 5.5 & 0.7 & 6 & 6 \\
\hline $\begin{array}{l}\text { How well the vet assessed the Johne's disease risk factors } \\
\text { on your farm }\end{array}$ & 0 & 2 & 5.5 & 0.7 & 6 & 6 \\
\hline Your level of involvement in the management plan decisions & 0 & 3 & 5.4 & 0.8 & 6 & 6 \\
\hline $\begin{array}{l}\text { Your understanding of the management options for Johne's } \\
\text { disease on your farm }\end{array}$ & 0 & 3 & 5.0 & 0.8 & 5 & 6 \\
\hline The interest the vet expressed in your opinion & 0 & 2 & 5.2 & 0.9 & 5 & 6 \\
\hline The amount of information you received from the vet & 0 & 1 & 5.2 & 0.9 & 5 & 6 \\
\hline How well the vet addressed all of your concerns & 1 & 3 & 5.5 & 0.7 & 6 & 6 \\
\hline The amount of time the vet spent with you & 1 & 3 & 5.5 & 0.7 & 6 & 6 \\
\hline Your overall experience with the vet during the RAMP ${ }^{3}$ & 0 & 4 & 5.6 & 0.6 & 6 & 6 \\
\hline
\end{tabular}

${ }^{1}$ Scored on a 6 -point Likert scale, with 1 being poor and 6 being excellent.

${ }^{2}$ Number of responses.

${ }^{3}$ Global satisfaction item.

els due to a priori reasoning. The univariable analyses only identified 1 variable that had an association with a $P<0.10$; herds that were enrolled in a milk-recording program had overall satisfaction rating scores that were 0.17 out of 6.0 less than herds not enrolled in a milkrecording program $(P=0.097 ; 95 \% \mathrm{CI}$ : $-0.37,0.03)$. Due to the lack of associations detected with the other demographic and herd information variables available in this study, a multivariable linear mixed model was not built.

\section{Knowledge Transfer in the AJDI}

There were no missing responses to the 14 knowledge-based questions in the questionnaires. The mean percentage of correct responses for all 3 infectious diseases (JD, BVD, and BLV) was $63.4 \%$ (95\% CI: $60.6 \%, 66.1 \%)$. The Shapiro-Wilk test statistics for the percentages of correct responses overall and for each of the 3 infectious diseases were not significant $(P>$ 0.2 ), indicating the assumptions of normality were not violated. Therefore, parametric statistical techniques were used. The infectious disease that had the highest percentage of correct responses was JD, with a mean percentage of $69.8 \%$ (95\% CI: 66.6, 72.9\%). Compared with JD knowledge responses, both BVD and BLV had significantly $(P<0.01$ for both) lower percentages of correct responses, with mean percentages of $60.7 \%$ (95\% CI: $56.3 \%, 65.1 \%)$ and $59.1 \%$ (95\% CI: $54.5 \%$, $63.7 \%$ ). A significant difference was not detected in JD knowledge based upon the preceding EC herd categorization $(P=0.90)$.

Participants were asked if the certified veterinarian discussed BLV or BVD during their preceding JD RAMP. Of the 133 dairy producers, 21.1\% indicated that the certified veterinarian discussed both BLV and BVD during the preceding RAMP $(\mathrm{n}=28), 36.1 \%$ indicated that the certified veterinarian discussed BLV but not BVD ( $\mathrm{n}=48)$, and $33.1 \%$ discussed BVD but not BLV $(\mathrm{n}=44)$. A significant difference was detected in BLV knowledge based upon whether or not the certified veterinarian discussed BLV during the preceding RAMP $(P<0.01)$. Of the dairy producers that discussed BLV during the preceding RAMP, the mean percentage of correct BLV responses was $71.3 \%$ (95\% CI: $65.3 \%, 77.2 \%)$. In contrast, the mean percentage of correct BLV responses was $52.2 \%$ (95\% CI: $46.2 \%$, $58.3 \%$ ) for those who did not discuss BLV during the preceding RAMP. This association was not affected by the length of time between the herd's preceding RAMP and the questionnaire (i.e., no interaction was found between the effect of discussing BLV during the preceding RAMP and timing of the questionnaire). A significant difference was not detected in BVD knowledge based upon whether or not the certified veterinarian discussed BVD during the preceding RAMP $(P=0.27)$.

Figure 1 depicts the distribution of responses to each of the knowledge-based questions in the questionnaire. For the questions about JD, the items with the highest proportions of producers responding correctly were related to age susceptibility of infection $(90.2 \%$ of producers responded correctly) and MAP transmission (94.7\% were correct). The JD items with the lowest proportions of producers responding correctly were related to individual cow diagnostics $(42.1 \%)$ and the clinical sign of diarrhea in JD (54.9\%). The responses to the questions about BVD indicated good understanding about the virus being able to cause abortion $(82.7 \%$ of producers responded correctly) and the efficacy of vaccination ( $81.2 \%$ were correct). However, there was a 


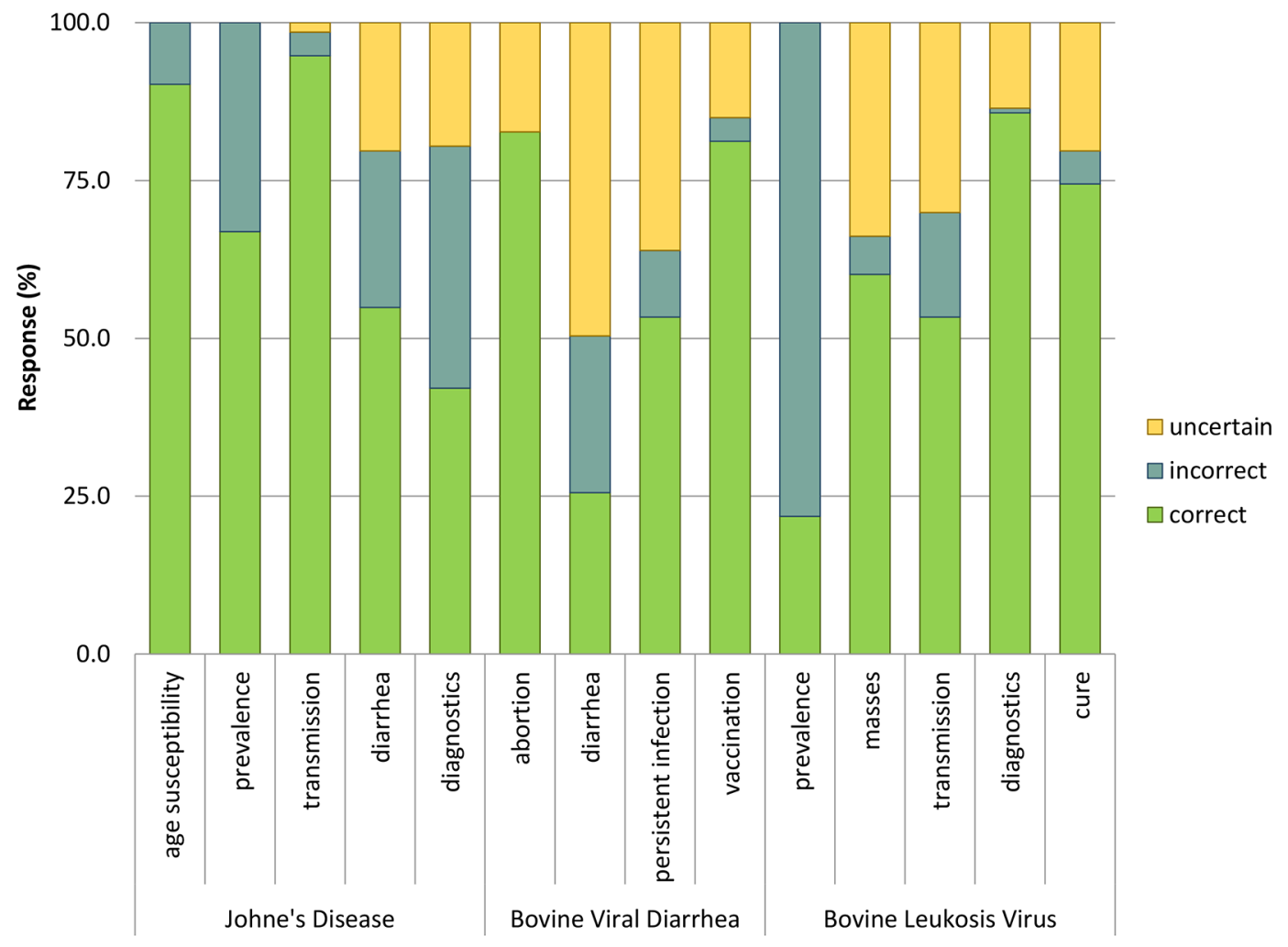

Figure 1. Distribution of responses to the knowledge questions in the producer satisfaction questionnaire for the risk assessment and management plan for 133 dairy producers in the Atlantic Johne's Disease Initiative.

lack of BVD knowledge about diarrhea as a clinical sign (49.6\% of producers responded that they were uncertain) and persistent infections (36.1\% were uncertain). For the questions about BLV, the responses indicated there was substantial uncertainty about the virus (proportions of producers with uncertain responses ranged from 13.5 to $33.8 \%$ ), and a majority $(78.2 \%$ ) of the producers underestimated the prevalence of leukosis in the Maritimes.

\section{DISCUSSION}

This study represented one of the methods used to evaluate the AJDI, which was designed to prevent and control JD in Atlantic Canada through the implementation of best management practices. To our knowledge, this is the first explorative quantitative study of satisfaction about the RAMP process in a JD control program. This work contributes to understanding producer satisfaction and knowledge transfer in the AJDI, which in turn improves understanding of other important outcomes including adherence.

Overall, producers were highly satisfied with the RAMP. High satisfaction was demonstrated using both the overall multi-item satisfaction measure and the global measure. There was a positive correlation detected between the overall satisfaction score and the global satisfaction score. This finding supports construct validity of the adapted satisfaction measure from the CSQ developed by Coe et al. (2010).

Unlike previous satisfaction studies in human and veterinary medicine (Coe et al., 2010; Shaw et al., 2012; Ritter et al., 2019), factors that influenced RAMPspecific producer satisfaction were not found in this study. In contrast to previous literature, client gender was not shown to have a significant effect on RAMPspecific satisfaction. It is of note that the ratio of male to female clients in this study was opposite to the ratio in the studies to develop and validate the CSQ and the VSSQ (Woodcock and Barleggs, 2005; Coe et al., 2010). Considering that both of those satisfaction measures were designed for companion animal medicine, satisfaction factors may be different due to differences in food animal production medicine and dairy producers. Other factors that had been shown to have significant associations with client satisfaction (e.g., client age, client education, and length of relationship with the veterinarian) were not available in this study. Therefore, we were unable to use them to further investigate construct validity or to describe the influence they had 
on RAMP-specific satisfaction. It is also possible that the satisfaction questionnaire items were not discerning enough to differentiate producers by RAMP-specific satisfaction and, consequently, factors that influenced their satisfaction. Additional research to investigate relationships with RAMP-specific producer satisfaction is recommended to support or refute the construct validity of the satisfaction measure used in this study and to better understand factors that influence RAMPspecific producer satisfaction.

In satisfaction questionnaires, multi-item satisfaction measures are often favored because solutions are more easily generated than from single satisfaction item measures (Hudak and Wright, 2000). Indeed, from the multiple satisfaction items in this questionnaire, problem areas in RAMP-specific producer satisfaction were identified. The RAMP item with the lowest satisfaction rating was the certified veterinarians' discussions of costs. This satisfaction item also had the highest number of "unable to assess" responses. Similar to the CSQ, the cost-related satisfaction item was framed in a manner that the producers should have been able to answer; therefore, these responses may represent a general lack of cost discussion between the certified veterinarian and the producer (Coe et al., 2010). It is also possible that the cost-related responses were influenced by AJDI covering the costs of veterinary fees to conduct RAMP, even though this satisfaction item was intended to measure producer satisfaction with discussion of costs related to JD and management recommendations. Regardless, it is concerning that cost discussions during the RAMP are not highly satisfactory or may not even be occurring. In previous studies on the attitudes of dairy producers toward JD control programs, producers were concerned with the overall economic impact that JD can have on a farm and identified financial cost as a key issue to increase their prioritization of JD prevention and control (Sorge et al., 2010). The RAMP item with the second lowest satisfaction rating was producer understanding of management options for JD on their farm. With the design of the AJDI as an RA-based control program, the successful implementation of management practices is key to reducing the effect and prevalence of JD in Atlantic Canada. In communication research, it is well documented that client-centered care is an effective approach and has been strongly associated with adherence (Kurtz et al., 2005; Silverman et al., 2013). Client-centered care encourages client participation, negotiation, and shared decision making (Shaw et al., 2016). It would be necessary for the producers to understand the management options to control JD on their farm to fully engage in shared decision making, and in turn be more likely to adhere to the management plan for their farm. Due to the identification of these 2 problem areas during the veterinary-administered RAMP, interventions can be developed to improve the communication of the certified veterinarians about costs and management options to control JD.

The producers in this study had moderate knowledge about JD. It is encouraging that most producers understood age susceptibility of MAP transmission and risk of MAP transmission even from cows that appear healthy. These items are related to best management practices focusing on internal biosecurity to prevent the spread of MAP within a herd. In contrast, more than half of the producers incorrectly thought that JD diagnostic tests on individual animals were highly sensitive. This misunderstanding could prevent the proper implementation of best management practices that focus on external biosecurity to prevent introducing or increasing the burden of MAP in a herd. Particularly for herds without JD, keeping MAP out of the herd would be preferred and more practical than trying to eradicate MAP once it had become established. Even with appropriate management, decreasing the prevalence of MAP in a herd is a process that takes years. Additional efforts should be made by the certified veterinarians to communicate information about JD diagnostics with the dairy producers.

Knowledge about JD was significantly higher than knowledge about BLV and BVD. For the majority of the herds in the study, the questionnaires were administered following the herd's second EC herd categorization. As such, most of the herds had sustained exposure to JD information through the AJDI, including 2 herd EC categorizations, 2 farm-specific RAMP with their certified veterinarian, and a full description of JD best management practices in the AJDI workbook left on farm following each RAMP. We did anticipate that BLV knowledge would also be moderate in the study, as herd and cow prevalence of BLV is very high in this geographic region and has been subject to previous research (Nekouei et al., 2015a). However, BLV knowledge was not significantly different from BVD knowledge in this study, even though BVD has been subject to much less research and discussion in this region.

Evidence of knowledge transfer during the RAMP was mixed in this study. The BVD knowledge scores were not found to differ based on whether or not the certified veterinarian discussed BVD during the preceding JD RAMP, whereas BLV knowledge scores were significantly higher among the dairy producers that discussed BLV during the preceding JD RAMP. It is possible that these findings were affected by misclassification bias; producers may have remembered incorrectly whether or not they discussed BVD or BLV during their preceding RAMP, and so their exposures were er- 
roneously categorized. This misclassification would bias the measure of knowledge transfer toward the null. If knowledge transfer during the RAMP was indeed poor, it would represent a communication failure. Regardless of the cause for the lack of evidence about knowledge transfer during the RAMP, improved communication skills of the certified veterinarians would be beneficial to enhance the measure of knowledge transfer. Additional research to further explore knowledge transfer during the RAMP is required to determine the degree of urgency required for this intervention.

In this study, a couple of limitations need to be considered. The representativeness of the study population may be limited to places with similar herd size, management, and JD prevalence. However, there was little evidence of selection bias, suggesting good internal validity of the study results. For example, the response rate was high, and the demographic and production characteristics of the study herds were similar to that of AJDI herds in total. Before extrapolation of study results to herds outside the region, consideration of comparability with the study population is recommended.

A second limitation was the variable length of time between a herd's preceding RAMP and the questionnaire. It is possible that responses to the questionnaire were affected by this limitation; for example, data quality could have suffered in some circumstances due to difficulties associated with producer recollection of their satisfaction with their preceding RAMP or whether or not they discussed BVD or BLV during their preceding RAMP. However, evidence was not found in the study to demonstrate an effect of questionnaire timing. For example, an association was not detected between questionnaire timing and producer satisfaction, and no interaction was found between the effect of discussing BLV during the preceding RAMP and timing of the questionnaire.

In spite of these limitations, the usefulness of an adapted CSQ as an outcome measure of producer satisfaction was demonstrated by this study. Further use and evaluation of adapted CSQ in other contexts of food animal production medicine or infectious disease control could enhance understanding of producer satisfaction and the effect of other factors such as adherence.

\section{CONCLUSIONS}

A RAMP-specific producer satisfaction questionnaire was successfully adapted from a companion animal appointment-specific questionnaire and used in a voluntary JD control program. Overall, producer satisfaction with the veterinary-administered RAMP process was high. The questionnaire items were useful from a programmatic standpoint in that they identified satis- faction items that required improvement during RAMP delivery, such as discussions of costs and producers' understanding of management options for JD. In addition, the questionnaire found there were knowledge gaps and issues with knowledge translation that should be addressed. Interventions to improve communication skills could support client-centered care, improve the certified veterinarians' communication about costs and management options to control JD, and in turn improve other important outcomes such as adherence. Further research in RAMP-specific producer satisfaction is recommended to expand the evaluation of construct validity of the questionnaire in different contexts and larger populations of both dairy producers and veterinarians and to identify factors that influence RAMP satisfaction.

\section{ACKNOWLEDGMENTS}

The project was funded by the Dairy Farmers of Prince Edward Island (Charlottetown, PE, Canada), Nova Scotia (Truro, NS, Canada), New Brunswick (Sussex, NB, Canada), and Newfoundland and Labrador (St. John's, NL, Canada); and Agriculture and Agri-Food Canada (Ottawa, ON, Canada) in conjunction with the Provinces of Prince Edward Island (Charlottetown, PE, Canada), Nova Scotia (Halifax, NS, Canada), New Brunswick (Fredericton, NB, Canada), and Newfoundland and Labrador (St. John's, NL, Canada) through the Canadian Agricultural Partnership. We thank the participating dairy producers for their involvement in the AJDI and this study. We also thank Jane Saunders-Jewell, survey administration, and Ricky Milton, data entry, both of University of Prince Edward Island, PE, Canada, for their hard work. The authors have not stated any conflicts of interest.

\section{REFERENCES}

Atreya, R., M. Bülte, G. Gerlach, R. Goethe, M. W. Hornef, H. Köhler, J. Meens, P. Möbius, E. Roeb, and S. Weiss. 2014. Facts, myths and hypotheses on the zoonotic nature of Mycobacterium avium subspecies paratuberculosis. Int. J. Med. Microbiol. 304:858-867. https://doi.org/10.1016/j.ijmm.2014.07.006.

Barker, R. A., H. W. Barkema, G. Fecteau, G. P. Keefe, and D. F. Kelton. 2012. Johne's disease control in Canada - Coordinated nationally, delivered provincially. Pages $41-46$ in 3rd ParaTB Forum. IDF Bull. No. 460. International Dairy Federation.

Bartlett, E. E., M. Grayson, R. Barker, D. M. Levine, A. Golden, and S. Libber. 1984. The effects of physician communications skills on patient satisfaction; recall, and adherence. J. Chronic Dis. 37:755764. https://doi.org/10.1016/0021-9681(84)90044-4.

Bauman, C. A., H. W. Barkema, J. Dubuc, G. P. Keefe, and D. F. Kelton. 2016. Identifying management and disease priorities of Canadian dairy industry stakeholders. J. Dairy Sci. 99:10194-10203. https://doi.org/10.3168/jds.2016-11057.

Bell, R. A., R. L. Kravitz, D. Thom, E. Krupat, and R. Azari. 2002. Unmet expectations for care and the patient-physician relation- 
ship. J. Gen. Intern. Med. 17:817-824. https://doi.org/10.1046/j $.1525-1497.2002 .10319 . x$.

Bragadóttir, H., and D. Reed. 2002. Psychometric instrument evaluation: The pediatric family satisfaction questionnaire. Pediatr. Nurs. 28:475-482.

Chiodini, R. J., W. M. Chamberlin, J. Sarosiek, and R. W. McCallum. 2012. Crohn's disease and the mycobacterioses: A quarter century later. Causation or simple association? Crit. Rev. Microbiol. 38:52-93. https://doi.org/10.3109/1040841X.2011.638273.

Coe, J. B., C. L. Adams, K. Eva, S. Desmarais, and B. N. Bonnett. 2010. Development and validation of an instrument for measuring appointment-specific client satisfaction in companion-animal practice. Prev. Vet. Med. 93:201-210. https://doi.org/10.1016/j prevetmed.2009.10.005.

Collins, M. T., V. Eggleston, and E. J. B. Manning. 2010. Successful control of Johne's disease in nine dairy herds: Results of a six-year field trial. J. Dairy Sci. 93:1638-1643. https://doi.org/10.3168/jds .2009-2664.

Dohoo, I. R., W. Martin, and H. Stryhn. 2009. Veterinary Epidemiologic Research. 2nd ed. VER Inc.

Hudak, P. L., and J. G. Wright. 2000. The characteristics of patient satisfaction measures. Spine 25:3167-3177. https://doi.org/10 .1097/00007632-200012150-00012.

Jackson, J. L., J. Chamberlin, and K. Kroenke. 2001. Predictors of patient satisfaction. Soc. Sci. Med. 52:609-620. https://doi.org/10 .1016/S0277-9536(00)00164-7.

Kanji, N., J. B. Coe, C. L. Adams, and J. R. Shaw. 2012. Effect of veterinarian-client-patient interactions on client adherence to dentistry and surgery recommendations in companion-animal practice. J. Am. Vet. Med. Assoc. 240:427-436. https://doi.org/ 10.2460/javma.240.4.427.

Kurtz, S. M., J. Silverman Dr, and J. Draper. 2005. Teaching and Learning Communication Skills in Medicine. 2nd ed. Radcliffe Publishing Ltd.

Larsen, D. E., and I. Rootman. 1976. Physician role performance and patient satisfaction. Soc. Sci. Med. 10:29-32. https://doi.org/10 .1016/0037-7856(76)90136-0

LeBlanc, S. J., K. D. Lissemore, D. F. Kelton, T. F. Duffield, and K. E. Leslie. 2006. Major advances in disease prevention in dairy cattle. J. Dairy Sci. 89:1267-1279. https://doi.org/10.3168/jds.S0022 -0302(06)72195-6.

Locker, D., and D. Dunt. 1978. Theoretical and methodological issues in sociological studies of consumer satisfaction with medical care. Soc. Sci. Med. 12:283-292.

MacDonald-Phillips, K. A., G. P. Keefe, J. VanLeeuwen, D. H. Shaw, and S. L. B. McKenna. 2021. Dairy producer satisfaction and knowledge transfer with the veterinary-administered risk assessment and management plan in a voluntary Johne's disease control program - Supplemental file. University of Prince Edward Island, Robertson Library's Data Repository. https://data.upei.ca/ islandora/object/researchdata\%3A679.

Nekouei, O., H. Stryhn, J. VanLeeuwen, D. Kelton, P. Hanna, and G. Keefe. 2015a. Predicting within-herd prevalence of infection with bovine leukemia virus using bulk-tank milk antibody levels. Prev. Vet. Med. 122:53-60. https://doi.org/10.1016/j.prevetmed.2015.10 .009 .

Nekouei, O., J. Vanleeuwen, J. Sanchez, D. Kelton, A. Tiwari, and G. Keefe. 2015b. Herd-level risk factors for infection with bovine leukemia virus in Canadian dairy herds. Prev. Vet. Med. 119:105-113. https://doi.org/10.1016/j.prevetmed.2015.02.025.

Nielsen, S. S. 2007. Danish control programme for bovine paratuberculosis. Cattle Pract. 15:161-168.

Ridge, S. E., I. M. Baker, and M. Hannah. 2005. Effect of compliance with recommended calf-rearing practices on control of bovine Johne's disease. Aust. Vet. J. 83:85-90. https://doi.org/10.1111/j .1751-0813.2005.tb12204.x.
Ritter, C., C. L. Adams, D. F. Kelton, and H. W. Barkema. 2019. Factors associated with dairy farmers' satisfaction and preparedness to adopt recommendations after veterinary herd health visits. J. Dairy Sci. 102:4280-4293. https://doi.org/10.3168/jds.2018-15825.

Roche, S. M., A. Jones-Bitton, M. Meehan, M. Von Massow, and D. F. Kelton. 2015. Evaluating the effect of focus farms on Ontario dairy producers' knowledge, attitudes, and behavior toward control of Johne's disease. J. Dairy Sci. 98:5222-5240. https://doi .org/10.3168/jds.2014-8765.

Shaw, J. R., C. L. Adams, B. N. Bonnett, S. Larson, and D. L. Roter. 2012. Veterinarian satisfaction with companion animal visits. J. Am. Vet. Med. Assoc. 240:832-841. https://doi.org/10.2460/ javma.240.7.832.

Shaw, J. R., G. E. Barley, K. Broadfoot, A. E. Hill, and D. L. Roter. 2016. Outcomes assessment of on-site communication skills education in a companion animal practice. J. Am. Vet. Med. Assoc. 249:419-432. https://doi.org/10.2460/javma.249.4.419.

Silverman, J., S. M. Kurtz, and J. Draper. 2013. Skills for Communicating with Patients. 3rd ed. CRC Press, Taylor \& Francis Group.

Sitzia, J., and N. Wood. 1997. Patient satisfaction: A review of issues and concepts. Soc. Sci. Med. 45:1829-1843. https://doi.org/ 10.1016/S0277-9536(97)00128-7.

Sorge, U., D. Kelton, K. Lissemore, A. Godkin, S. Hendrick, and S. Wells. 2010. Attitudes of Canadian dairy farmers toward a voluntary Johne's disease control program. J. Dairy Sci. 93:1491-1499. https://doi.org/10.3168/jds.2009-2447.

Sweeney, R. W., M. T. Collins, A. P. Koets, S. M. McGuirk, and A. J. Roussel. 2012. Paratuberculosis (Johne's disease) in cattle and other susceptible species. J. Vet. Intern. Med. 26:1239-1250. https: //doi.org/10.1111/j.1939-1676.2012.01019.x.

Tiwari, A., J. A. VanLeeuwen, S. L. B. McKenna, G. P. Keefe, and H. W. Barkema. 2006. Johne's disease in Canada: Part I: Clinical symptoms, pathophysiology, diagnosis, and prevalence in dairy herds. Can. Vet. J. 47:874-882.

Wassink, G. J., T. R. N. George, J. Kaler, and L. E. Green. 2010. Footrot and interdigital dermatitis in sheep: Farmer satisfaction with current management, their ideal management and sources used to adopt new strategies. Prev. Vet. Med. 96:65-73. https://doi.org/10 .1016/j.prevetmed.2010.06.002.

Wolf, R., H. W. Barkema, J. De Buck, and K. Orsel. 2015. Factors affecting management changes on farms participating in a Johne's disease control program. J. Dairy Sci. 98:7784-7796. https://doi .org/10.3168/jds.2015-9610.

Wolf, R., F. Clement, H. W. Barkema, and K. Orsel. 2014. Economic evaluation of participation in a voluntary Johne's disease prevention and control program from a farmer's perspective-The Alberta Johne's Disease Initiative. J. Dairy Sci. 97:2822-2834. https://doi .org/10.3168/jds.2013-7454.

Woodcock, A., and D. Barleggs. 2005. Development and psychometric validation of the veterinary service satisfaction questionnaire (VSSQ). J. Vet. Med. A Physiol. Pathol. Clin. Med. 52:26-38. https://doi.org/10.1111/j.1439-0442.2004.00676.x.

\section{ORCIDS}

Karen A. MacDonald-Phillips @ https://orcid.org/0000-0001-5218 $-564 \mathrm{X}$

Greg P. Keefe $\odot$ https://orcid.org/0000-0003-2356-7344

John VanLeeuwen (1) https://orcid.org/0000-0003-3340-0337

J. McClure @ https://orcid.org/0000-0002-7932-7967

Shawn L. B. McKenna ๑ https://orcid.org/0000-0002-9699-1464 